\title{
Colorectal Serrated Polyp With Stromal Changes: An Interobserver Agreement Study
}

\author{
Daniela S. Allende ${ }^{\mathrm{a}}$, Rish K. Pai ${ }^{\mathrm{b}}$, Hao Xie ${ }^{\mathrm{c}}$, Xiuli Liu ${ }^{\mathrm{d}, \mathrm{e}}$
}

\begin{abstract}
Background: Prolapse-related changes have been shown to be a confounding factor leading to misdiagnosis of serrated polyps in the rectum. Recent data on perineurial-like stromal proliferation in some sessile serrated polyps (SSPs) also highlights this issue.
\end{abstract}

Methods: Fifty-four consecutive serrated polyps with stromal changes from 42 patients were collected by one pathologist during a 2-month period (July and August of 2014). In addition, 20 cases of serrated polyps with perineurial-like stromal proliferation from 20 patients were retrieved from our pathology database from 2003 to 2010. The polyps were re-reviewed by three gastrointestinal pathologists for stromal changes, basal crypt distortion, and final classification into hyperplastic polyp (HP) or SSP. Final interpretation was correlated to clinicopathologic features and the presence of synchronous SSP and adenoma. In addition, the interobserver agreement on the stromal changes and basal crypt distortion in these polyps was determined.

Results: Upon histology review, all polyps showed stromal changes evident by at least one pathologist. Among the 74 polyps evaluated, a consensus diagnosis of HP and consensus diagnosis of SSP were reached in 39 and 11 polyps respectively. The overall interobserver agreement among three pathologists was moderate (kappa value 0.49 , $95 \%$ confidence interval (CI) 0.32 - 0.66). A consensus diagnosis could not be reached in the remaining 24 polyps (32.4\%). The SSPs were larger, were more often located in the right colon, and occurred more frequently in women when compared to HPs ( $7.3 \pm 5.3$ vs. 4.5 $\pm 2.7 \mathrm{~mm}, \mathrm{P}=0.019 ; 72.7 \%$ vs. $7.7 \%, \mathrm{P}=0.000014$; and $77.7 \%$ vs. $30.7 \%, \mathrm{P}=0.019)$ but with comparable patient age $(54.7 \pm 10.6$ years vs. $60.7 \pm 11.4, \mathrm{P}=0.12$ ). The SSPs were associated with higher risk of concurrent SSP in other parts of the colon $(27.3 \%$ vs. $0 \%, \mathrm{P}=$ 0.008 ) but not with the presence of concurrent adenoma (45.4\% vs.

Manuscript submitted September 30, 2019, accepted October 16, 2019

aDepartment of Anatomic Pathology, Cleveland Clinic, Cleveland, OH, USA bepartment of Laboratory Medicine and Pathology, Mayo Clinic Arizona, Scottsdale, AZ, USA

'Division of Medical Oncology, Mayo Clinic, Rochester, MN, USA

dDepartment of Pathology, Immunology and Laboratory Medicine, University of Florida, Gainesville, FL, USA

eCorresponding Author: Xiuli Liu, Department of Pathology, Immunology and Laboratory Medicine, University of Florida, Gainesville, FL 32610, USA. Email: xiuliliu@ufl.edu

doi: https://doi.org/10.14740/gr1230
$69.6 \%, \mathrm{P}=1$ ). Among the 24 unclassifiable serrated polyps, 11 were interpreted by two reviewers as SSP and 13 interpreted by two reviewers as HP; the former group were more likely right-sided $(45.4 \%$ vs. $15.4 \%, \mathrm{P}=0.046$ ), but size of the polyps and age of the patients were comparable. Stromal changes (prolapse and perineurial-like) were agreed upon by all three pathologists in 33 (44.6\%, of 74) polyps and these included 15 with prolapse changes and 18 perineurial-like stromal changes. Among those 15 with prolapse changes, 14 (93.3\%) were agreed upon by three pathologists to be HP; in contrast, only four $(22.2 \%$, of 18$)$ with perineurial-like stromal changes were diagnosed as HP by all three pathologists $(\mathrm{P}=0.00008)$. Of 74 polyps, 16 were found to have basal crypt distortion involving more than one crypt and eight of them (50\%) carried a final interpretation of SSP by all three pathologists and two (12.5\%) HP.

Conclusions: Even though serrated polyps with stromal changes can be challenging, up to $67.7 \%$ of them could be readily classified as SSP or HP. The remaining unclassifiable serrated polyps with stromal changes may represent a heterogeneous group. Prolapse changes support the diagnosis of HP while basal crypt distortion in more than one crypt supports the diagnosis of SSP.

Keywords: Sessile serrated polyp; Hyperplastic polyp; Prolapse; Perineurial-like stromal proliferation; Colorectum

\section{Introduction}

The serrated neoplasia pathway is increasingly recognized as a mechanism of sporadic colorectal cancer (CRC). Between $20 \%$ and $30 \%$ of all CRCs arise from the serrated pathway [1]. The serrated neoplasia pathway includes methylation of $\mathrm{CpG}$ island methylator phenotype (CIMP), mutation in the BRAF oncogene, and epigenetic silencing DNA mismatch repair genes [2]. Histologically, serrated lesions of the colon and rectum are characterized by sawtooth architecture of the crypts. Precursor serrated lesions to CRC include hyperplastic polyps (HPs), sessile serrated polypadenomas (SSPs) and traditional serrated adenomas (TSAs). The 2012 US Multi Society Task Force updated surveillance recommendations for serrated lesions on the basis that size $>10 \mathrm{~mm}$, histology, and location (proximal to the sigmoid colon) are risk factors that might be associated with higher risk of CRC $[3,4]$.

Serrated polyps include three major categories: HP, SSP, and TSA. The distinction between HP and SSP can be difficult. 
The histologic diagnosis of SSP is primarily based on histologic architectural features [5-7]. The overall interobserver agreement on the diagnosis of SSP had been reported as moderate $(\mathrm{k}=0.56)$ [8]. Two types of stromal changes (i.e. prolapse changes and perineurial-like stromal changes) have been observed in serrated polyp [9-11] and post another hurdle to the diagnosis. The presence of prolapse-related changes causes diagnostic challenge in rectal serrated polyps and is a source of misdiagnosis in rectal serrated polyps [11]. However, interobserver agreement on the stromal changes in colorectal serrated polyps has not been reported. In addition, the significance of specific subtypes of stromal changes in the diagnosis of colorectal serrated polyps has not been reported previously.

The aims of this study are to assess the interobserver agreement in diagnosing colorectal serrated polyps with stromal changes and in subtyping stromal changes in serrated polyp and to determine whether specific subtypes of stromal change are associated with the diagnosis of colorectal serrated polyps.

\section{Materials and Methods}

\section{Study case selection and histology review}

This study was approved by the institutional review board at Cleveland Clinic, and is complied with the ethical standards for human/animal study. Fifty-four consecutively diagnosed serrated polyps with stromal changes were collected from 42 patients by one pathologist (XL) during a 2-month period (July and August of 2014). In addition, 20 cases of serrated polyps with perineurial-like stromal proliferation from $20 \mathrm{pa}-$ tients were retrieved from our pathology database from 2003 to 2010 . The perineurial-like stromal proliferation was well characterized by immunohistochemistry and was consistent with prior publications [11]. The polyps were re-reviewed by three gastrointestinal pathologists and final interpretation in this study rendered based on histomorphology $[5,6,12,13]$. Subclassification of HP into microvesicular, goblet cell-rich, and mucin poor was not attempted as there is no clinical management difference for these subtypes of HP. The polyps were also assessed for the absence or presence of stromal changes: absence of stromal changes is defined as normal lamina propria surrounding serrated glands, prolapse-related changes are defined as smooth muscle proliferation in the lamina propria and entrapment and distortion of crypts [11], and perineuriallike stromal changes are defined as a band-like spindled stroma widely separating serrated crypts [10]. In most cases, these spindle cells surrounded serrated crypts, did not extend into the submucosa, and did not show pleomorphism, necrosis, or mitotic activity. In addition, the reviewing pathologists were asked to review if the cryptal architectural abnormalities involve one crypt or more than one crypt. The pathologists were blinded to endoscopic size, endoscopic appearance, and location of the polyps. For each polyp, each pathologist was required to give one of the following two diagnoses: HP and SSP.

The patient demographics and clinical data were obtained by reviewing electronic medical records. The location of the polyp was based on the information provided by the en- doscopist. The polyp was considered proximal if it was located at or proximal to the splenic flexure and distal if distal to the splenic flexure. The size of the polyp was either based on the information provided by the endoscopist or measured from the histology section whenever there was a discrepancy.

\section{Statistical analysis}

Clinicopathologic characteristics were compared between patients with HP and patients with SSP using the Student's $t$-test, $\chi^{2}$-test or Wilcoxon rank sum test. Fleiss' kappa was used to assess the interobserver agreement on the diagnosis, type of stromal changes, and basal crypt distortion. A kappa value $<$ $0,0.01-0.20,0.21-0.40,0.41-0.60,0.61-0.80$, and $0.81-$ 1.0 is considered poor, slight, fair, moderate, substantial, and almost perfect agreement, respectively [14]. A P value $<0.05$ was considered statistically significant. Statistical analysis was performed using R 2.15.2 (R Development Core Team, 2012, Vienna, Austria).

\section{Results}

A total of 74 polyps from 62 patients ( 34 men and 28 women) with a mean age of 61 years ( \pm standard deviation (SD) 11) were reviewed in this study. Among the 74 polyps evaluated, a consensus diagnosis was reached in 50 polyps $(67.6 \%)$. These include 39 HPs and 11 SSPs. The overall interobserver agreement among three pathologists was moderate (kappa value $0.49,95 \%$ confidence interval (CI) 0.32 - 0.66). The SSPs were larger, were more often located in the right colon, and occurred more frequently in women when compared to HPs $(7.3 \pm 5.3$ vs. $4.5 \pm 2.7 \mathrm{~mm}, \mathrm{P}=0.019 ; 72.7 \%$ vs. $7.7 \%, \mathrm{P}=0.000014$; and $77.7 \%$ vs. $30.7 \%, \mathrm{P}=0.019)$ but with comparable patient age $(54.7 \pm 10.6$ years vs. $60.7 \pm 11.4, \mathrm{P}=0.12)($ Table 1$)$. The SSPs were associated with higher risk of concurrent SSP in other parts of the colon $(27.3 \%$ vs. $0 \%, \mathrm{P}=0.008)$ but not with the presence of concurrent adenoma $(45.4 \%$ vs. $69.6 \%, \mathrm{P}=1)$ (Table 1). Examples of polyp with a consensus diagnosis of HP and SSP were illustrated in Figure 1a, b.

A consensus diagnosis could not be reached in the remaining 24 polyps (32.4\%). Among these 24 unclassifiable serrated polyps, 11 were interpreted by two reviewers as SSP and 13 interpreted by two reviewers as HP; the former group were more likely right-sided ( $45.4 \%$ vs. $15.4 \%, \mathrm{P}=0.046)$, but size of the polyp $(5.5 \pm 1.5$ vs. $4.9 \pm 2.3, \mathrm{P}=0.44)$, age of the patients $(59.3 \pm 11$ vs. $58.1 \pm 9.6, \mathrm{P}=0.78)$, and the right-sided location rate $(45.4 \%$ vs. $15 \%, \mathrm{P}=0.36)$ between these two subcategories were comparable. One example of unclassifiable serrated polyp with stromal change was illustrated in Figure 1c.

All polyps were evaluated for the absence or presence of stromal changes by three pathologists. Upon histology review, all polyps showed stromal changes evident by at least one pathologist. The overall interobserver agreement on the subtype of stromal changes among three pathologists was fair (kappa value $0.37,95 \%$ CI $0.25-0.48)$. Stromal changes were agreed upon by all three pathologists in 33 (44.6\%, of 74$)$ polyps. 
Table 1. Demographics, Polyp Characteristics and Rates of Concurrent SSP and Adenoma in Other Parts of the Colon in Patients With Consensus SSP and HP Demonstrating Stromal Changes

\begin{tabular}{llll} 
& Consensus SSP & Consensus HP & P value \\
\hline Female gender (\%) & 77.7 & 30.7 & 0.019 \\
Age (in years), mean (SD) & $54.7(10.6)$ & $60.7(11.4)$ & 0.12 \\
Size (in mm), mean (SD) & $7.3(5.3)$ & $4.5(2.7)$ & 0.019 \\
Right colon (\%) & 72.7 & 7.7 & 0.000014 \\
Current SSP in other parts of the colon (\%) & 27.3 & 0 & 0.008 \\
Current adenoma in other parts of the colon (\%) & 45.4 & 69.6 & 1 \\
\hline
\end{tabular}

SSP: sessile serrated polyps; HP: hyperplastic polyp; SD: standard deviation.

These included 15 with prolapse changes and 18 perineuriallike stromal changes; consensus HP was rendered in 54.5\% of these polyps and SSP in $18.2 \%$ (Table 2). The subtype of stromal changes was associated with the consensus diagnosis (Table 2). More specifically, among those 15 with prolapse changes, 14 (93.3\%) were agreed upon by three pathologists to be HP; in contrast, only four $(22.2 \%$, of 18$)$ with perineuriallike stromal changes were diagnosed as HP by all three pathologists $(\mathrm{P}=0.00008)$. In the remaining polyps with stromal changes, 21 were HP, five were SSP, eight were diagnosed with HP by two pathologists and SSP by one pathologist, and seven SSP by two pathologists and HP by one pathologist.

All polyps were evaluated for the absence or presence of basal crypt distortion including serration and lateral branching at the base by three pathologists. The overall interobserver agreement on basal crypt distortion among three pathologists was fair (kappa value 0.23 , 95\% CI 0.11 - 0.35). In addition, the three pathologists were asked to assess whether the basal crypt distortion was limited to one crypt or more than one crypt. The consensus interpretation of basal crypt distortion was associated with the consensus polyp diagnosis (Table 2). More specifically, of 74 polyps, four cases were found to harbor no basal crypt distortion and three of them were diagnosed as HP and one as unclassifiable serrated polyp; 16 were found to have basal crypt distortion involving more than one crypt and eight of them $(50 \%)$ carried a final interpretation of SSP by all three pathologists and two (12.5\%) HP; and one polyp was found to have basal crypt distortion involving only one crypt and it was diagnosed as HP by three pathologists. Thirteen polyps were found to have basal crypt distortion by two pathologists and three of them $(23.1 \%)$ were diagnosed as SSP and four $(30.7 \%) \mathrm{HP}$.

\section{Discussion}

The serrated neoplasia pathway has been implicated in the pathogenesis of up to $1 / 3$ of sporadic CRC cases. A total of $20 \%$ to $30 \%$ of all CRCs arise from the serrated pathway [1]. Accurate histologic diagnosis of serrated polyps plays a significant role in the management of patients $[3,4]$. Despite of all efforts of immunohistochemical markers and molecular characterization, the diagnosis of SSP in clinical practice is based on histological assessment of architectural abnormalities [8, $13,15]$. Challenges in the diagnosis of serrated polyps in the colorectum include suboptimal orientation of polyps during embedding process and the presence of prolapse-related stromal changes $[11,16]$. A previous study revealed prolapse-related changes in the rectal serrated polyps as a confounder for misdiagnosing SSPs in the rectum leading to overdiagnosing HPs as SSPs in about $42 \%$ of rectal serrated polyps [11]. Another study reported a reclassification rate of left-sided SSPs to HP in $15(57.7 \%$, of 26) cases [17] upon histology review of the originally diagnosed left-sided SSPs. In addition to prolapse-related stromal changes, there is another type of stromal changes in SSP, namely perineurial-like stromal proliferation $[10,18]$. However, interobserver agreement in the diagnosis of colorectal serrated polyp with stromal changes has not been studied. Further interobserver agreement in subtyping stromal changes in colorectal serrated polyps has not been reported.
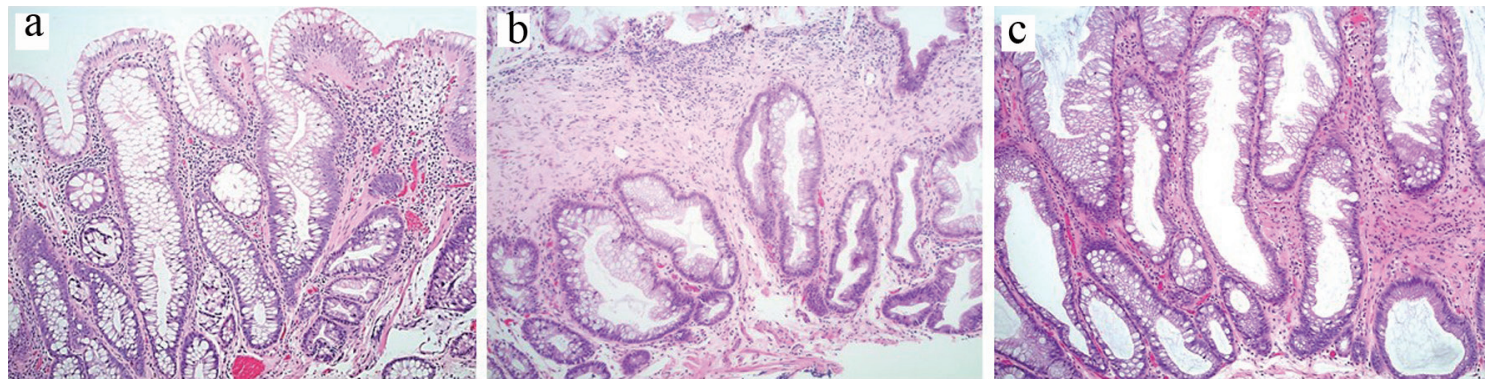

Figure 1. (a) Polyp with prolapse changes and a consensus diagnosis of hyperplastic polyp. (b) Polyp with perineurial-like stromal changes and a consensus diagnosis of sessile serrated polyp. (c) Polyp with stromal changes, unclassifiable: two pathologists diagnosed this polyp as sessile serrated polyp and one pathologist as hyperplastic polyp. 
Despite the above mentioned difficulties, the significance of specific type stromal changes, namely prolapse-related stromal change and perineurial-like stromal proliferation in the diagnosis of colorectal polyps, has not been previously reported.

In this study, we examined the interobserver agreement in the diagnosis of colorectal serrated polyps with stromal changes. We found that a consensus diagnosis of HP and SSP was reached in 50 (of $74,67.6 \%$ ) polyps with a moderate interobserver agreement (paired kappa value from 0.42 to 0.56 ). The proportion of consensus diagnosis of serrated polyp is slightly higher in comparison to a previous study which reported a consensus diagnosis reached in 60 out of 109 (55\% vs. $67.6 \%$, P $=0.03)[8]$ consecutively collected colorectal serrated polyps with or without stromal changes. A consensus in the identification of prolapse-related stromal changes and perineurial-like stromal proliferation was reached in $33(44.6 \%$, of 74$)$ polyps. The consensus interpretation of prolapse-related polyp by three pathologists was associated with a consensus interpretation of HP. More specifically, among those 15 with prolapse changes, $14(93.3 \%)$ were agreed upon by three pathologists to be HP; in contrast, four $(22.2 \%$, of 18$)$ with perineurial-like stromal changes were diagnosed as HP by all three pathologists $(\mathrm{P}=0.00008)$.

In our study, despite the fact that the pathologists were blinded to the clinical and endoscopic information at the time of their histologic evaluation, the consensus SSPs were larger, were more often located in the right colon, occurred more frequently in women when compared to HPs but with comparable patient age. The SSPs were associated with higher risk of concurrent SSP in other parts of the colon but not with the presence of concurrent adenoma. This finding was consistent with previous studies [9].

Currently, a single unequivocal architecturally distorted, dilated, and/or horizontally branched crypt in a serrated polyp is considered to be sufficient for a diagnosis of SSP [7, 13]. Thus in this study, we evaluated the presence of basal crypt distortion (dilatation and horizontally branching) in 74 polyps with stromal changes. Our results revealed that basal crypt distortion involving more than one crypt can only be identified by three pathologists in 16 polyps (of 76, 21\%). This feature, if agreed upon by three pathologists, was associated with a diagnosis of SSP. More specifically, eight of them $(50 \%$, of 16$)$ carried a final interpretation of SSP by all three pathologists. The results suggest that the presence of more than one crypt remains a critical histologic finding to diagnose SSP in serrated polyps with stromal changes particularly if the distorted crypt is located distant from the stromal changes.

Many strengths can be highlighted in this study. This is the first study to evaluate interobserver agreement on diagnosing colorectal serrated polyps with stromal changes. All 74 polyps were considered to have stromal changes by at least one pathologist upon histological review. In addition, three pathologists were gastrointestinal pathology fellowship trained, practiced in a pathology department with large volume of polyps, and had more than 5 years of experience in practicing pathology. All of them also showed strong interest and published articles in serrated polyps $[8,19]$. The study also examined whether the basal crypt distortion (dilatation and horizontally branching) involves one or more than one crypt. 
The study harbors some limitations including a relatively small number of polyps. The HPs were not further sub-classified into microvesicular HP, goblet cell-rich HP, and mucinpoor HP. However, this sub-classification of HP has no impact on clinical management of these patients. Although this study revealed that the SSPs were associated with higher risk of concurrent SSP in other parts of the colon $(27.3 \%$ vs. $0 \%$, $\mathrm{P}=0.008$ ) but not with the presence of concurrent adenoma $(45.4 \%$ vs. $69.6 \%, \mathrm{P}=1)$, it did not investigate if SSP is associated with subsequent risk of SSP or CRC. Further, the clinical significance of those unclassifiable serrated polyp in this study is not clear.

In conclusion, this study revealed that even though serrated polyps with stromal changes can be challenging, up to $67.6 \%$ of them could be readily classified based on histologic examination alone as SSP or HP. The presence of prolapserelated stromal change supports a diagnosis of HP and the presence of basal crypt distortion in more than one crypt supports a diagnosis of SSP. The remaining unclassifiable serrated polyps with stromal changes may represent a heterogeneous group.

\section{Acknowledgments}

None to declare.

\section{Financial Disclosure}

None to declare.

\section{Conflict of Interest}

None to declare.

\section{Informed Consent}

Not applicable.

\section{Author Contributions}

DSA contributed to pathology review and manuscript drafting; XL contributed to study design and pathology review; RKP was involved in pathology review and manuscript critical review; and HX was involved in data analysis and manuscript critical review.

\section{References}

1. Leggett B, Whitehall V. Role of the serrated pathway in colorectal cancer pathogenesis. Gastroenterology 2010;138:2088-2100.

2. Snover DC. Update on the serrated pathway to colorectal carcinoma. Hum Pathol. 2011;42(1):1-10.

3. Schreiner MA, Weiss DG, Lieberman DA. Proximal and large hyperplastic and nondysplastic serrated polyps detected by colonoscopy are associated with neoplasia. Gastroenterology. 2010;139(5):1497-1502.

4. Hiraoka S, Kato J, Fujiki S, Kaji E, Morikawa T, Murakami $T$, Nawa $T$, et al. The presence of large serrated polyps increases risk for colorectal cancer. Gastroenterology. 2010;139(5):1503-1510, 1510 e1501-1503.

5. Torlakovic E, Snover DC. Serrated adenomatous polyposis in humans. Gastroenterology. 1996;110(3):748-755.

6. Torlakovic E, Skovlund E, Snover DC, Torlakovic G, Nesland JM. Morphologic reappraisal of serrated colorectal polyps. Am J Surg Pathol. 2003;27(1):65-81.

7. Pai RK, Bettington M, Srivastava A, Rosty C. An update on the morphology and molecular pathology of serrated colorectal polyps and associated carcinomas. Mod Pathol. 2019;32(10):1390-1415.

8. Gonzalo DH, Lai KK, Shadrach B, Goldblum JR, Bennett AE, Downs-Kelly E, Liu X, et al. Gene expression profiling of serrated polyps identifies annexin A10 as a marker of a sessile serrated adenoma/polyp. J Pathol. 2013;230(4):420-429.

9. Pai RK, Hart J, Noffsinger AE. Sessile serrated adenomas strongly predispose to synchronous serrated polyps in non-syndromic patients. Histopathology. 2010;56(5):581588.

10. Pai RK, Mojtahed A, Rouse RV, Soetikno RM, Kaltenbach T, Ma L, Arber DA, et al. Histologic and molecular analyses of colonic perineurial-like proliferations in serrated polyps: perineurial-like stromal proliferations are seen in sessile serrated adenomas. Am J Surg Pathol. 2011;35(9):1373-1380.

11. Huang CC, Frankel WL, Doukides T, Zhou XP, Zhao W, Yearsley MM. Prolapse-related changes are a confounding factor in misdiagnosis of sessile serrated adenomas in the rectum. Hum Pathol. 2013;44(4):480-486.

12. Snover DC, Ahnen DJ, Burt RW, Odze RD. Serrated polyps of the colon and rectum and serrated polyposis. In: Bosman FT et al. editors. WHO Classification of Tumours of the Digestive System. Lyon, France: IARC Press; 2010. p. 160-165.

13. Rex DK, Ahnen DJ, Baron JA, Batts KP, Burke CA, Burt RW, Goldblum JR, et al. Serrated lesions of the colorectum: review and recommendations from an expert panel. Am J Gastroenterol. 2012;107(9):1315-1329; quiz 1314, 1330 .

14. Landis JR, Koch GG. The measurement of observer agreement for categorical data. Biometrics. 1977;33(1):159174.

15. Cui M, Awadallah A, Liu W, Zhou L, Xin W. Loss of Hes 1 Differentiates Sessile Serrated Adenoma/Polyp From Hyperplastic Polyp. Am J Surg Pathol. 2016;40(1):113-119.

16. Choi EY, Appelman HD. A Historical Perspective and Expose on Serrated Polyps of the Colorectum. Arch Pathol Lab Med. 2016;140(10):1079-1084.

17. Chetty R, Wang LM, Gill P, East JE, Leedham S. Leftsided sessile serrated polyps/adenomas. Hum Pathol. 2013;44(9):1959-1960. 
18. Agaimy A, Stoehr R, Vieth M, Hartmann A. Benign serrated colorectal fibroblastic polyps/intramucosal perineuriomas are true mixed epithelial-stromal polyps (hybrid hyperplastic polyp/mucosal perineurioma) with frequent BRAF mutations. Am J Surg Pathol. 2010;34(11):1663-
1671.

19. Jackson WE, Achkar JP, Macaron C, Lee L, Liu X, Pai RK, Lopez R, et al. The significance of sessile serrated polyps in inflammatory bowel disease. Inflamm Bowel Dis. 2016;22(9):2213-2220. 